\title{
An electrographic AV optimization for the maximum integrative atrioventricular and ventricular resynchronization in CRT
}

Jie Li ${ }^{1,2+}$, Yuegang Wang ${ }^{4 \dagger}$, Jingting Mai ${ }^{3 \dagger}$, Shilan Chen ${ }^{1,2+}$, Menghui Liu ${ }^{1,2}, C^{\prime}$ Chen Su ${ }^{1,2}$, Xumiao Chen ${ }^{1,2}$, Huiling Huang ${ }^{1,2}$, Yuedong Ma ${ }^{1,2}$, Chong Feng ${ }^{1,2}$, Jingzhou Jiang ${ }^{1,2}$, Jun Liu ${ }^{1,2}$, Jiangui He ${ }^{1,2}$, Anli Tang ${ }^{1,2}$, Yugang Dong ${ }^{1,2}$, Xiaobo Huang ${ }^{4}$, Yangxin Chen ${ }^{3^{*}}$ and Lichun Wang ${ }^{1 *}$

\begin{abstract}
Background: Atrioventricular (AV) delay could affect $A V$ and ventricular synchrony in cardiac resynchronization therapy (CRT). Strategies to optimize AV delay according to optimal AV synchrony ( $A V_{\text {opt-AV }}$ ) or ventricular synchrony $\left(A V_{\text {opt- }}\right)$ would potentially be discordant. This study aimed to explore a new AV delay optimization algorithm guided by electrograms to obtain the maximum integrative effects of $A \bigvee$ and ventricular resynchronization (opt-AV).
\end{abstract}

Methods: Forty-nine patients with CRT were enrolled. $A V_{\text {opt-AV }}$ was measured through the Ritter method. $A V_{\text {opt- }}$ was obtained by yielding the narrowest $Q R S$. The opt-AV was considered to be $A V_{\text {opt-AV }}$ or $A V_{\text {opt- }}$ when their difference was $<20 \mathrm{~ms}$, and to be the $A V$ delay with the maximal aortic velocity-time integral between $A V_{\text {opt-AV }}$ and $A V_{\text {opt- }}$ when their difference was $>20 \mathrm{~ms}$.

Results: The results showed that sensing/pacing $A V_{\text {opt-AV }}\left(S_{A V} V_{\text {opt-AV }} / \mathrm{PAV}_{\text {opt-AV }}\right)$ were correlated with atrial activation time $\left(P_{\text {end-As }} / P_{\text {end-Ap }}\right)(P<0.05)$. Sensing $/$ pacing $A V_{\text {opt- }}\left(S A V_{\text {opt- } V} / P A V_{\text {opt- }}\right)$ was correlated with the intrinsic AV conduction time (As-Vs/Ap-Vs) $(P<0.01)$. The percentages of patients with more than 20 ms differences between $S A V_{\text {opt-AV/ }}$ $\mathrm{PAV}_{\text {opt-AV }}$ and $S A V_{\text {opt- }} / \mathrm{PAV}$ opt- $\mathrm{V}$ were $62.9 \%$ and $57.1 \%$, respectively. Among them, opt-AV was linearly correlated with $\mathrm{SAV}_{\text {opt-AV }} / \mathrm{PAV}_{\text {opt-AV }}$ and SAV $\mathrm{opt}_{\mathrm{V}-\mathrm{V}} / \mathrm{PAV}_{\text {opt-V. }}$ The sensing opt-AV (opt-SAV) $=0.1 \times \mathrm{SAV}_{\text {opt-AV }}+0.4 \times \mathrm{SAV}_{\text {opt-V }}+70 \mathrm{~ms}$ $\left(R^{2}=0.665, P<0.01\right)$ and the pacing opt-AV $($ opt-PAV $)=0.25 \times \mathrm{PAV}_{\text {opt-AV }}+0.5 \times \mathrm{PAV}_{\text {opt }-\mathrm{V}}+30 \mathrm{~ms}\left(\mathrm{R}^{2}=0.560, P<0.01\right)$.

Conclusion: The SAV opt-AV/PAV opt-AV and $S A V_{\text {opt- } V} / \mathrm{PAV}_{\text {opt- }}$ were correlated with the atrial activation time and the intrinsic AV conduction interval respectively. Almost half of the patients had a $>20$ ms difference between SAV ${ }_{\text {opt-AV/ }}$ PAV $\mathrm{opt}-\mathrm{AV}_{\text {a }}$ and $S A V_{\text {opt- } \mathrm{V}} / \mathrm{PAV}_{\text {opt- } \mathrm{V}}$. The opt-AV could be estimated based on electrogram parameters.

Keywords: AV delay, Atrioventricular synchrony, Ventricular synchrony, Cardiac resynchronization therapy

*Correspondence: chenyx39@mail.sysu.edu.cn; wanglich@mail.sysu.edu.cn ${ }^{\dagger}$ Jie Li, Yuegang Wang, Jingting Mai, and Shilan Chen have contributed equally to this work

${ }^{1}$ Department of Cardiology, The First Affiliated Hospital of Sun Yat-Sen University, No. 58, Zhongshan 2nd Rd, Guangzhou 510080, Guangdong, People's Republic of China

${ }^{3}$ Department of Cardiology, Sun Yat-Sen Memorial Hospital of Sun YatSen University, No.107, Yanjianxi Rd, Guangzhou 510080, Guangdong, People's Republic of China

Full list of author information is available at the end of the article

\section{Background}

Cardiac resynchronization therapy (CRT) is a milestone therapy in advanced congestive heart failure for its ability in decreasing symptoms, improving quality of life and exercise capacity, and reducing hospitalization and mortality in selected patients with heart failure $[1,2]$. However, up to $30-45 \%$ of patients do not respond to CRT therapy $[1,3]$. Among them, almost $50 \%$ cases have suboptimal atrioventricular (AV) timing [4]. 
The AV interval can affect AV and ventricular synchrony simultaneously. However, improving AV and ventricular dyssynchrony is the underlying therapeutic mechanism of CRT. To obtain optimal AV synchrony, an AV interval is required to ensure that the left ventricle (LV) only contracts after completion of left atrial (LA) contraction [5]. At this time, the optimal AV interval is subject to the atrial activation time. If there is an inter/ intra-atrial conduction delay, a relatively long AV interval is required for delayed LA contraction. Furthermore, ventricular resynchronization is maximally achieved through the narrowest QRS, which is obtained by optimal fusion between intrinsic atrioventricular activation and paced activation [6, 7]. Therefore, the optimal AV interval should coincide with the intrinsic AV conduction interval for obtaining optimal ventricular synchrony.

As a result, in some cases, the optimal AV delay for maintaining AV synchrony may be quite different from that for maintaining ventricular synchrony. For example, in patients with a long PR interval but normal atrial conduction, optimal ventricular resynchronization (the narrowest QRS) would require a longer AV delay, while this might lead to suboptimal AV resynchronization since a normal atrial conduction requires a relatively short $\mathrm{AV}$ delay.

In this study, the AV intervals were optimized according to optimal AV synchrony $\left(\mathrm{AV}_{\text {opt-AV }}\right)$ and optimal ventricular synchrony $\left(\mathrm{AV}_{\mathrm{opt}-\mathrm{V}}\right)$. The relationships between $\mathrm{AV}_{\text {opt-AV }}$ and the atrial activation time, $\mathrm{AV}_{\text {opt- } \mathrm{V}}$ and the intrinsic $A V$ interval, $A V_{\text {opt-AV }}$ and $A V_{\text {opt-V }}$ were further investigated. The aim was to study the difference between $A V_{\text {opt-AV }}$ and $A V_{\text {opt-V }}$, and to propose a novel AV optimized algorithm guided only by the intrinsic AV interval and the atrial activation time to obtain the maximal integrative effects of $\mathrm{AV}$ and ventricular resynchronization.

\section{Methods}

\section{Study population}

This was a multicenter, nonrandomized study, that enrolled patients aged 18 or older, who had been implanted with CRT defibrillators with standard criteria (NYHA classes II-IV; ejection fraction $\leq 35 \%$; sinus rhythm; left bundle branch block with $\mathrm{QRS} \geq 130 \mathrm{~ms}$ ). Patients were excluded from the study if they had congenital heart diseases, valve repair or replacement surgeries, atrial tachyarrhythmias or frequent atrial or ventricular ectopy, and second/third degree of AV block. The measurements were performed at least 1 month after CRT implantation to reduce the effect of the operation. The protocol of this study was approved by the institutional review boards of the participating hospitals, and all patients gave written informed consent.

\section{Study procedures General data}

Clinical data, such as demographics (age, sex, etc.), etiology, heart failure status before CRT device implantation (NYHA classes, left ventricular end-diastolic diameter, left ventricular end-systolic diameter, ejection fraction), medications, CRT device data (model number and date of implant), and the location of the right atrium and LV electrodes were collected before the study procedure.

\section{Electrocardiogram and device electrograms}

The patient's intrinsic electrocardiogram (ECG) was recorded after the device marker recording showed atrial sensing (As) and ventricular sensing (Vs) by programming the lower rate to $50 / 40 \mathrm{bpm}$ and the sensed AV delay to $300 / 350 \mathrm{~ms}$. Heart rate, QRS duration, PR interval, device-recorded intrinsic $\mathrm{AV}$ conduction interval (As-Vs), and atrial activation time (duration from As to the end of the $\mathrm{P}$ wave $\left.\left[\mathrm{P}_{\text {end-As }}\right]\right)$ were sequentially measured. Then the lower rate was programmed to $10 \mathrm{bpm}$ higher than the intrinsic heart rate. The interval from atrial pacing (Ap) to Vs (Ap-Vs), and the duration from Ap to the end of the $\mathrm{P}$ wave $\left(\mathrm{P}_{\text {end-Ap }}\right)$ were also measured sequentially at least 10 times (Fig. 1).

\section{Measurement of AV delay according to the optimal atrioventricular synchrony}

The Ritter method was originally developed for AV delay setting to achieve optimal AV synchrony in patients with a complete AV block and preserved LV function [8]. Its aim was to maximize LV filling (including maximizing the role of LA contraction) and to minimize pre-systolic mitral regurgitation by ensuring that the left ventricular contraction starts soon after the completion of the left atrial contraction. Then, it and its analogue became the gold standard for AV delay optimization in CRT [9]. In this study, we used the Ritter method to optimize $\mathrm{AV}_{\text {opt-AV }}$. In brief, mitral flow velocity and surface ECG were simultaneously recorded. A short and a long $\mathrm{AV}$ delay $\left(\mathrm{AV}_{\text {short }} / \mathrm{AV}_{\text {long }}\right)$ were programmed, and the relevant intervals from the pacing spike $(\mathrm{Q})$ to the end of the Doppler mitral inflow A wave $\left(\mathrm{QA}_{\text {short }} / \mathrm{QA}_{\text {long }}\right)$ were measured. $A V_{\text {opt-AV }}$ was calculated as follows: $A V_{\text {opt-AV }}=\mathrm{AV}_{\text {long }}+\mathrm{QA}_{\text {long }}-\mathrm{QA}_{\text {short }}[8,10]$. In this study, the $\mathrm{VV}$ intervals were always kept at $0 \mathrm{~ms}$.

\section{Measurement of the AV delay according to the optimal ventricular synchrony}

The narrower the QRS duration was, the more synchronous the ventricle was. Simultaneous 12-lead ECGs were recorded when the sensing/pacing AV delays (SAV/PAV) were programmed to values from $70 \mathrm{~ms}$ to an AV delay 

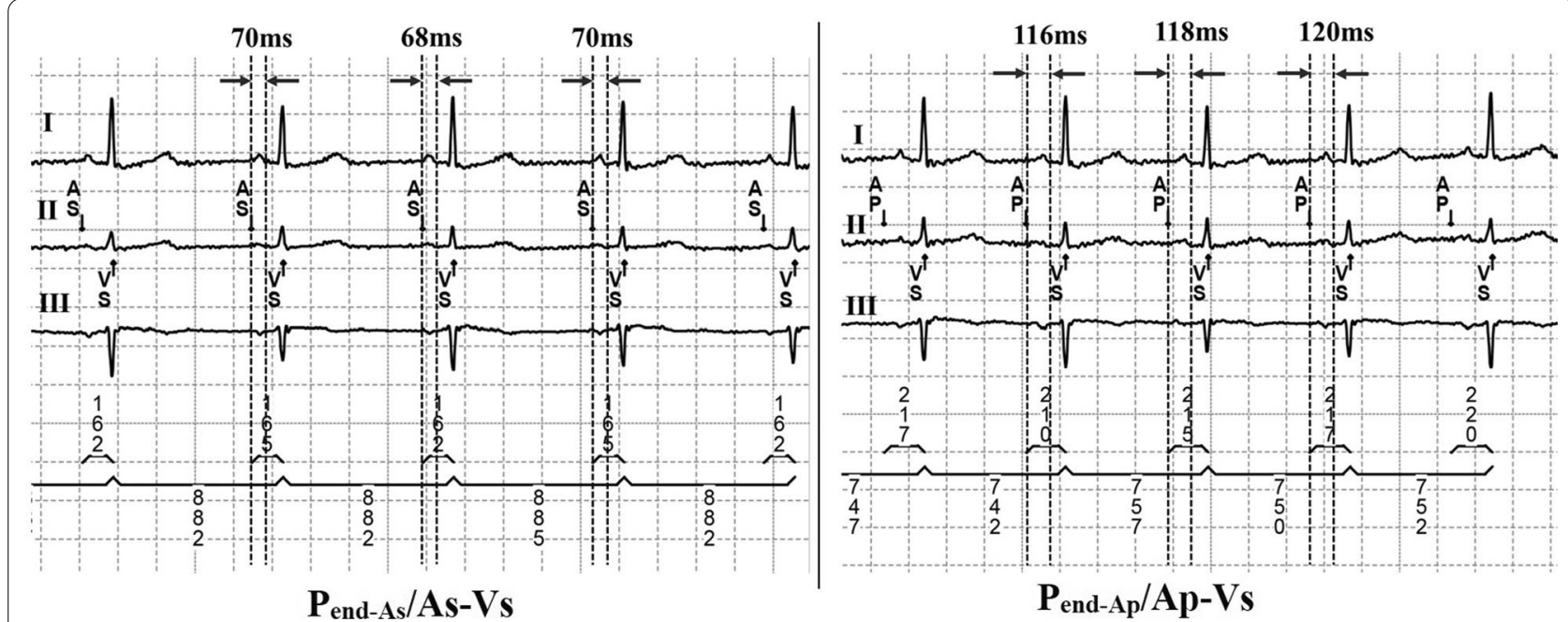

Fig. 1 Measurement of atrial activation time and intrinsic atrioventricular conduction time. Left panel: atrial activation time $\left(P_{\text {end-As }}\right)$ and intrinsic atrioventricular conduction time (As-Vs) at the time of atrial sensing. Right panel: atrial activation time $\left(\mathrm{P}_{\text {end-Ap }}\right)$ and intrinsic atrioventricular conduction time (Ap-Vs) at the time of atrial pacing. $A_{s}$ : atrial sensing; $A_{p}$ : atrial pacing; $V_{s}$ : ventricular sensing

of $40 \mathrm{~ms}$ less than the intrinsic As-Vs/Ap-Vs by 10-ms steps in a random order. The durations of the QRS were automatically calculated by the built-in software in the
ECG machine. The SAV/PAV delays with the narrowest QRS duration were considered sensing/pacing $A V_{\text {opt-V }}$ $\left(\mathrm{SAV}_{\text {opt-V }} / \mathrm{PAV}_{\text {opt-V }}\right)($ Fig. 2).

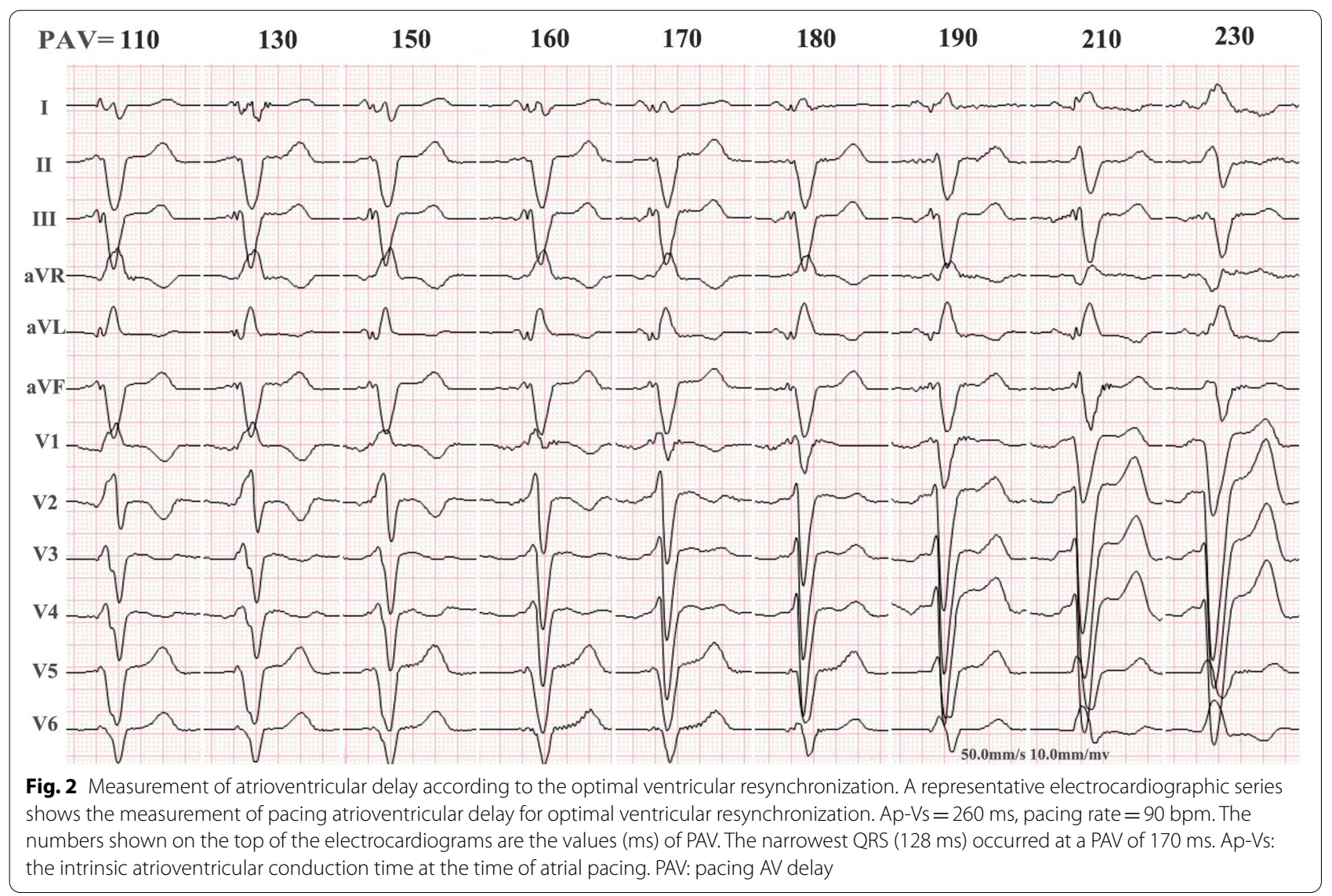




\section{Determination of the optimal AV delay}

When sensing/pacing $\mathrm{AV}_{\text {opt-AV }}\left(\mathrm{SAV}_{\text {opt-AV }} / \mathrm{PAV}_{\text {opt-AV }}\right)$ with $\mathrm{SAV}_{\text {opt-V}} / \mathrm{PAV}_{\text {opt-V}}$ was compared, if the difference in values (D-values) was $<20 \mathrm{~ms}, \mathrm{SAV}_{\text {opt-AV }} / \mathrm{PAV}_{\text {opt-AV }}$ and $\mathrm{SAV}_{\text {opt-V}} / \mathrm{PAV}_{\text {opt-V }}$ were regarded as not different $[11,12]$, and were considered as the optimal sensing/pacing AV delay (opt-SAV/opt-PAV). If the D-values were $\geq 20 \mathrm{~ms}$, the aortic velocity-time integral (AoVTI) was measured by continuous wave Doppler recordings from $\mathrm{SAV}_{\text {opt-AV}} /$ $\mathrm{PAV}_{\text {opt-AV }}$ to $\mathrm{SAV}_{\text {opt-V }} / \mathrm{PAV}_{\text {opt-V }}$ by 10 -ms steps. SAV/PAV with the maximum AoVTI was considered as the optSAV/opt-PAV. All measurements of echocardiographic data were averaged from 9 to 12 consecutive cardiac beats.

\section{Statistical analysis}

Continuous variables that were normally distributed are shown as the mean $\pm \mathrm{SD}$. Non-normally distributed variables are shown as medians and interquartile ranges. The data were analyzed with IBM SPSS software version 20.0 for Windows (IBM Inc., Armonk, NY, USA). The pairedsamples t-test or two related-samples Wilcoxon rank sum tests were used for between-group statistical analysis according to the evaluation of a normal distribution. Regression analysis and Pearson's correlation coefficient were performed to evaluate the correlations. $P<0.05$ was defined as statistically significant.

\section{Results}

\section{Patient population}

A total of 49 patients were enrolled in this study from three hospitals (the First Affiliated Hospital of Sun Yat-Sen University, Sun Yat-Sen Memorial Hospital of Sun Yat-Sen University, and Nanfang Hospital of Nanfang Medical University) from July 2017 to May 2020. The clinical characteristics of the patients are shown in Table 1 . The majority of the patients (35/49) were men. The mean intrinsic PR interval and QRS duration were $185.88 \pm 38.20$ (115-275) $\mathrm{ms}$ and $166.29 \pm 21.34$ (146237) ms, respectively. The atrial electrodes were placed in the right atrial appendage in all patients.

\section{Relationship between $\mathrm{AV}_{\text {opt-Av }}$ and atrial activation time}

$\mathrm{AV}_{\text {opt-AV }}$ was successfully determined in 35 patients by the Ritter method. Regression analysis showed that $\mathrm{SAV}_{\text {opt-AV }}$ was significantly correlated with $\mathrm{P}_{\text {end-As }}$ $\left(\mathrm{SAV}_{\text {opt-AV }}=0.80 \times \mathrm{P}_{\text {end-As }}+50 \mathrm{~ms}, \mathrm{R}^{2}=0.467, P<0.01\right)$. A similar result was also found between $\mathrm{PAV}_{\mathrm{opt}-\mathrm{AV}}$ and $\mathrm{P}_{\text {end-Ap }}\left(\mathrm{PAV}_{\text {opt-AV }}=0.70 \times \mathrm{P}_{\text {end-Ap }}+70 \mathrm{~ms}, \quad \mathrm{R}^{2}=0.221\right.$, $P<0.05)$.
Table 1 Clinical characteristics of the patients involved in the study

\begin{tabular}{|c|c|}
\hline Age, years & $67.25 \pm 9.37$ \\
\hline Male/female & $35 / 14$ \\
\hline \multicolumn{2}{|l|}{ Etiology, n (\%) } \\
\hline Ischemic heart disease & $20(40.8)$ \\
\hline Dilated myocardiopathy & $24(49.0)$ \\
\hline Noncompaction of ventricular myocardium & $4(8.2)$ \\
\hline Other & $1(2.0)$ \\
\hline \multicolumn{2}{|l|}{ NYHA class, n (\%) } \\
\hline$\|$ & $13(28.9)$ \\
\hline III & $29(64.4)$ \\
\hline IV & $4(8.9)$ \\
\hline \multicolumn{2}{|l|}{ Medication, n (\%) } \\
\hline Beta-blocker & $42(93.3)$ \\
\hline ACEI/ARB & $27(60.0)$ \\
\hline MRA & $44(97.8)$ \\
\hline ARNI & $18(40.0)$ \\
\hline$I_{f}$-channel inhibitor & $3(6.7)$ \\
\hline Diuretics & $4(8.9)$ \\
\hline Digitalis & $3(6.7)$ \\
\hline \multicolumn{2}{|l|}{ UCG } \\
\hline LVEF (\%) & $28.66 \pm 4.94$ \\
\hline LVEDD (mm) & $70.42 \pm 10.29$ \\
\hline $\operatorname{LVESD}(\mathrm{mm})$ & $59.13 \pm 10.94$ \\
\hline PA pressure $(\mathrm{mmHg})$ & $38.45 \pm 6.98$ \\
\hline Diastolic mitral regurgitation, n (\%) & $31(68.9)$ \\
\hline \multicolumn{2}{|l|}{ ECG characteristics } \\
\hline Intrinsic heart rate (bpm) & $68.21 \pm 11.76$ \\
\hline PR interval (ms) & $185.88 \pm 38.20$ \\
\hline QRS duration (ms) & $166.29 \pm 21.34$ \\
\hline \multicolumn{2}{|l|}{ Location of LV lead, n (\%) } \\
\hline \multicolumn{2}{|l|}{ Short axis } \\
\hline Lateral/posterolateral & $32(65.3)$ \\
\hline Anterolateral & $13(25.5)$ \\
\hline Posterior & $4(8.2)$ \\
\hline Anterior & 0 \\
\hline \multicolumn{2}{|l|}{ Long axis } \\
\hline Basic & $16(32.7)$ \\
\hline Middle & $33(67.3)$ \\
\hline Apical & 0 \\
\hline \multicolumn{2}{|l|}{ Device electrogram characteristics } \\
\hline As-Vs (ms) & $200.02 \pm 34.26$ \\
\hline Ap-Vs (ms) & $258.33 \pm 45.46$ \\
\hline$P_{\text {end-As }}(\mathrm{ms})$ & $86.08 \pm 22.68$ \\
\hline$P_{\text {end-Ap }}(m s)$ & $136.84 \pm 23.92$ \\
\hline
\end{tabular}

NYHA: New York heart association; ACEl: Angiotensin-converting-enzyme inhibitor; ARB: Angiotensin II receptor antagonist; MRA: Aldosterone receptor

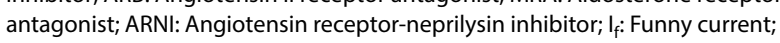
UCG: Ultrasonic cardiogram; LVEF: left ventricular ejection fraction; LVEDD: left ventricular end diastolic diameter; LVESD: left ventricular end systolic diameter; PA: Pulmonary artery; ECG: Electrocardiogram; As-Vs: Intrinsic atrioventricular conduction time at the time of atrial sensing; Ap-Vs: Intrinsic atrioventricular conduction time at the time of atrial pacing; $P_{\text {end-As }}$ : Atrial activation time at the 
Table 1 (continued)

time of atrial sensing; $P_{\text {end-Ap }}$ : Atrial activation time at the time of atrial pacing

Table 2 The percentage of patients with more than 20 ms differences between $A V_{\text {opt-AV }}$ and $A V_{\text {opt- } V}$

\begin{tabular}{|c|c|c|}
\hline & $\begin{array}{l}\text { Patients with directly } \\
\text { measured AV } \\
(n=35)\end{array}$ & $\begin{array}{l}\text { Patients with } \\
\text { calculated } \\
\mathrm{AV}^{*} \\
(\mathrm{n}=49)\end{array}$ \\
\hline SAV & 22 (62.9\%) & 22 (44.9\%) \\
\hline PAV & $20(57.1 \%)$ & $29(59.2 \%)$ \\
\hline SAV and PAV & $17(48.6 \%)$ & 17 (34.7\%) \\
\hline
\end{tabular}

*The Sensing/Pacing $\mathrm{AV}_{\text {opt-AV }}$ and $\mathrm{AV}_{\text {opt-V }}$ were calculated according to the following formulae:

$\mathrm{SAV}_{\text {opt-AV }}=0.80 \times \mathrm{P}_{\text {end-As }}+50 \mathrm{~ms}$

$\mathrm{SAV}_{\text {opt }-\mathrm{V}}=0.60 \times A \mathrm{As}-\mathrm{Vs}+15 \mathrm{~ms}$

$\mathrm{PAV}_{\text {opt-AV }}=0.70 \times \mathrm{P}_{\text {end-Ap }}+70 \mathrm{~ms}$

$\mathrm{PAV}_{\text {opt }-\mathrm{V}}=0.60 \times \mathrm{Ap}-\mathrm{Vs}+40 \mathrm{~ms}$

\section{Relationship between $\mathrm{AV}_{\text {opt-v }}$ and intrinsic $\mathrm{AV}$ conduction and $Q R S$ duration}

$\mathrm{SAV}_{\text {opt-V}} / \mathrm{PAV}_{\text {opt-V }}$ was achieved through the narrowest QRS duration in all 49 patients. $\mathrm{SAV}_{\text {opt-V }} / \mathrm{PAV}_{\text {opt- }}$ $\mathrm{V}$ was significantly correlated with As-Vs/Ap-Vs $\left(\mathrm{SAV}_{\text {opt-V }}=0.60 \times \mathrm{As}-\mathrm{Vs}+15 \mathrm{~ms}, \mathrm{R}^{2}=0.456, P<0.01\right.$; $\left.\mathrm{PAV}_{\text {opt-V }}=0.60 \times \mathrm{Ap}-\mathrm{Vs}+40 \mathrm{~ms}, \mathrm{R}^{2}=0.417, P<0.01\right)$, but there was no significant correlation with the intrinsic QRS duration (both $P>0.5$ ).

\section{Difference between $A V_{\text {opt-Av }}$ and $A V_{\text {opt-V }}$}

In the 35 patients, in which $\mathrm{SAV}_{\text {opt-AV }} / \mathrm{PAV}_{\text {opt-AV }}$ and $\mathrm{SAV}_{\text {opt-V }} / \mathrm{PAV}_{\text {opt-V }}$ were successfully directly measured simultaneously, no significant correlations were found between $\mathrm{SAV}_{\text {opt-AV }}$ and $\mathrm{SAV}_{\text {opt-V}}$, or between PAV $\mathrm{Ppt}_{\text {-AV }}$ and $P_{\text {opt-V }}$ (both $P>0.05$ ). If $>20-\mathrm{ms}$ D-values between $\mathrm{SAV}_{\text {opt-AV}} / \mathrm{PAV}_{\text {opt-AV }}$ and $\mathrm{SAV}_{\text {opt-V}} / \mathrm{PAV}_{\text {opt- }}$ $\mathrm{V}$ were considered as meaningful differences, 22/35 (62.9\%) patients had a $>20-\mathrm{ms}$ difference in SAV and 20/35 (57.1\%) had a > 20-ms difference in PAV. Among them, $17 / 35$ (48.6\%) patients had a $>20$-ms difference in both SAV and PAV. Moreover, if $\mathrm{SAV}_{\text {opt-AV }} / \mathrm{PAV}_{\text {opt- }}$ $\mathrm{AV}$ and $\mathrm{SAV}_{\text {opt-V}} / \mathrm{PAV}_{\text {opt-V }}$ were obtained by calculation according to their relationship with $\mathrm{P}_{\text {end-As }} / \mathrm{P}_{\text {end-Ap }}$ and As-Vs/Ap-Vs, respectively, > 20-ms D-values were found in 22/49 (44.9\%) patients in SAV, in 29/49 (59.2\%) patients in PAV, and in 17/49 (34.7\%) patients in both SAV and PAV (Table 2).
Relationships of opt-SAV/opt-PAV with $\mathrm{SAV}_{\text {opt-AV }} / \mathrm{PAV}_{\text {opt-AV }}$ and SAV $_{\text {opt-v }} /$ PAV $_{\text {opt-V }}$

Opt-SAV/opt-PAV was considered according to the maximum AoVTI when the D-values between $\mathrm{SAV}_{\text {opt-AV }} / \mathrm{PAV}_{\text {opt-AV }}$ and $\mathrm{SAV}_{\text {opt-V}} /$ $P A V_{\text {opt-V }}$ were $>20 \mathrm{~ms}$. Regression analysis showed that opt-SAV $=0.1 \times \mathrm{SAV}_{\text {opt-AV }}+0.4 \times \mathrm{SAV}_{\text {opt } \mathrm{V}}+70 \quad \mathrm{~ms}$ $\left(\mathrm{R}^{2}=0.665, \quad P<0.01\right) \quad$ and $\quad$ that $\mathrm{PAV}=0.25 \times \mathrm{PAV}_{\text {opt-AV }}+0.5 \times \mathrm{PAV}_{\text {opt-V}}+30 \quad \mathrm{~ms}$ $\left(\mathrm{R}^{2}=0.560, P<0.01\right)$. The relative higher coefficient of determination $\left(R^{2}\right)$ in these equations indicated that the actual values and the calculated values of the opt-SAV/ opt-PAV were highly related (Fig. 3).

\section{Echocardiographic evaluation of opt-SAV/opt-PAV}

The difference in haemodynamics was evaluated by echocardiographic measurement of AoVTI. In the cases with $>20$ ms D-values between $\mathrm{SAV}_{\text {opt-AV }}$ and SAV $\mathrm{Spt-V}_{\text {, }}$ the AoVTI on opt-SAV was significantly greater than the AoVTI on $\mathrm{SAV}_{\text {opt-v }}$ (opt-SAV $-\mathrm{SAV}_{\text {opt-V }}=1.52 \pm 0.22 \mathrm{~cm}$, $P<0.001)$, and was not less than that on SAVopt-AV (opt-SAV $-\mathrm{SAV}_{\text {opt-AV }}=0.89 \pm 0.82 \quad \mathrm{~cm}, \quad P=0.290$ ). Furthermore, the AoVTI on opt-PAV was also significantly greater than that on $\mathrm{PAV}_{\text {opt-AV }}$ (opt$\left.\mathrm{PAV}-\mathrm{PAV}_{\text {opt-AV }}=2.47 \pm 0.80 \quad \mathrm{~cm}, \quad P=0.006\right) \quad$ and $\mathrm{PAV}_{\text {opt } \mathrm{V}} \quad$ (opt-PAV $-\mathrm{PAV}_{\text {opt } \mathrm{V}}=0.76 \pm 0.30 \mathrm{~cm}$, $P=0.021)$.

\section{Discussion}

AV delay has effects on both AV and ventricular resynchronization simultaneously in CRT, and could be optimized according to the optimal AV and ventricular synchrony respectively. This study showed that $\mathrm{SAV}_{\text {opt-AV }} / \mathrm{PAV}_{\text {opt-AV }}$ were related to the atrial activation time $\left(\mathrm{P}_{\text {end-As }} / \mathrm{P}_{\text {end-Ap }}\right)$, and $\mathrm{SAV}_{\text {opt-V }} / \mathrm{PAV}_{\text {opt-V }}$ were related to the intrinsic atrioventricular conduction interval (As-Vs/Ap-Vs). However, nearly 50\% of patients showed a significant difference between $\mathrm{SAV}_{\text {opt-AV}} /$ $\mathrm{PAV}_{\text {opt-AV }}$ and $\mathrm{SAV}_{\text {opt-V}} / \mathrm{PAV}_{\text {opt-V }} \quad$ (D-values $\left.>20 \mathrm{~ms}\right)$. At this time, opt-SAV/opt-PAV optimized according to the maximal AoVTI were linearly correlated with $\mathrm{SAV}_{\text {opt-AV }} / \mathrm{PAV}_{\text {opt-AV }}$ and $\mathrm{SAV}_{\text {opt-V}} / \mathrm{PAV}_{\text {opt-V}}$, and had significantly improved haemodynamics. Therefore, the optimal AV delay in CRT could be considered as $\mathrm{SAV}_{\text {opt-AV}} / \mathrm{PAV}_{\text {opt-AV }}$ or $\mathrm{AV}$ opt-V $/ \mathrm{PAV}_{\text {opt-V }}$ if the D-values were $<20 \mathrm{~ms}$, or it could be achieved by formulas (opt$\mathrm{SAV}=0.1 \times \mathrm{SAV}_{\text {opt-AV }}+0.4 \times \mathrm{SAV}_{\text {opt } \mathrm{V}}+70 \mathrm{~ms} ; \quad$ opt$\left.\mathrm{PAV}=0.25 \times \mathrm{PAV}_{\text {opt-AV }}+0.5 \times \mathrm{PAV}_{\text {opt } \mathrm{V}}+30 \mathrm{~ms}\right)$ if the D-values were $>20 \mathrm{~ms}$. The AV optimized algorithm with the maximal integrative effects of $\mathrm{AV}$ and ventricular resynchronization is shown in Fig. 4. In this algorithm, the required parameters were just the atrial activation 


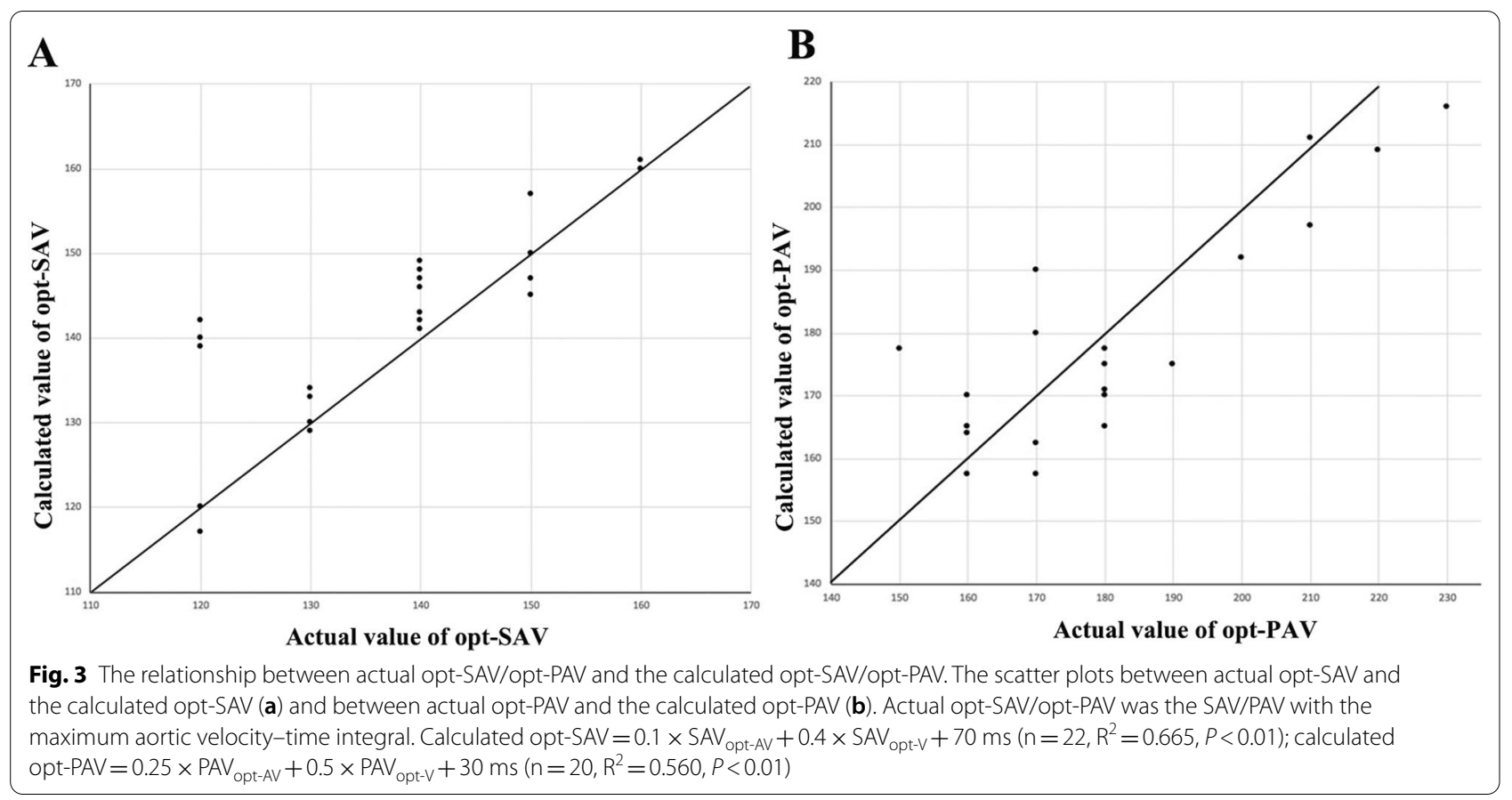

time (Pend-As/Pend-Ap) and the intrinsic atrioventricular conduction interval (As-Vs/Ap-Vs), which could be measured over several minutes during device interrogation. Therefore, it was easy to perform and special equipment was not required.

Optimal atrioventricular synchrony is a mechanical status in which the onset of LV contraction only coincides with the end of LA contraction and yields the longest diastolic filling time and a fully active filling phase. This is also the principle underlying the Ritter method and its analogues for the optimization of AV delay [9]. Previous studies have shown that this type of AV delay is related to the atrial conduction time [13]. In this study, we defined this AV delay for optimal atrioventricular synchrony as $A V_{\text {opt-AV }}$. Using the duration from As/Ap to the relevant end of the $\mathrm{P}$ wave $\left(\mathrm{P}_{\text {end-As }} / \mathrm{P}_{\text {end-Ap }}\right)$ as the atrial activation time, the results showed that $\mathrm{SAV}_{\text {opt-AV }} / \mathrm{PAV}_{\text {opt-AV }}$ were linearly correlated with $\mathrm{P}_{\text {end-As }} / \mathrm{P}_{\text {end-Ap }}$. Similar results were also found by Jones et al. [14], who suggested that $\mathrm{SAV}=\mathrm{P}_{\text {end-As }}+40 \mathrm{~ms}$ and $\mathrm{PAV}=\mathrm{P}_{\text {end-Ap }}+30 \mathrm{~ms}$. We did not find any significant differences when comparing these two equations with our formulas (both $P>0.05$, data not shown).

The duration of QRS is a marker of ventricular synchrony. Many studies have shown that the extent of a decrease in QRS duration is related to clinical improvement and reverse remodeling of the LV in CRT $[7,15,16]$. Achieving the narrowest QRS duration was first applied in VV optimization $[17,18]$. This was further used in AV optimization to maximize the LV global contractile function $[12,13,19]$ because the narrowest QRS duration could be obtained by maximal fusion between intrinsic atrioventricular activation and paced activation $[6$, 7]. Therefore, the AV delay for optimal ventricular synchrony according to the narrowest QRS duration should be correlated with the intrinsic AV conduction interval. In this study, we defined the AV delay for the narrowest QRS as $\mathrm{SAV}_{\text {opt- } \mathrm{V}} / \mathrm{PAV}_{\text {opt-V}}$, and found that they were correlated with intrinsic AV conduction (As-Vs/Ap-Vs).

However, in patients with CHF, the intrinsic AV conduction interval and the atrial activation time are not always proportional. In our patients, we could not find a significant correlation between As-Vs/Ap-Vs and $\mathrm{P}_{\text {end-As }} /$ $\mathrm{P}_{\text {end-Ap }}$ (data not shown). Therefore, the AV delay, which was optimized according to maximal AV synchrony and was correlated with the atrial activation time, did not always coincide with AV delay optimized according to the maximal ventricular synchrony, which was correlated with intrinsic AV conduction. In this study, no significant correlations were found between $\mathrm{SAV}_{\text {opt-AV }} / \mathrm{PAV}_{\text {opt- }}$ $\mathrm{AV}$ and $\mathrm{SAV}_{\text {opt-V}} / \mathrm{PAV}_{\text {opt-V}}$, and almost $50 \%$ of patients showed $>20$-ms differences between $\mathrm{SAV}_{\text {opt-AV }} / \mathrm{PAV}_{\text {opt-AV }}$ and $\mathrm{SAV}_{\text {opt-V }} / \mathrm{PAV}_{\text {opt-V}}$. These findings indicated that the AV delay optimized only according to the optimal AV synchrony or ventricular synchrony was not optimal in approximately half of the patients with CRT. In fact, some studies showed that the narrowest QRS complex in CRT was not always associated with the maximal improvement of cardiac contractive function [13]. Sometimes the AV delay optimized by the Ritter method is not as useful 


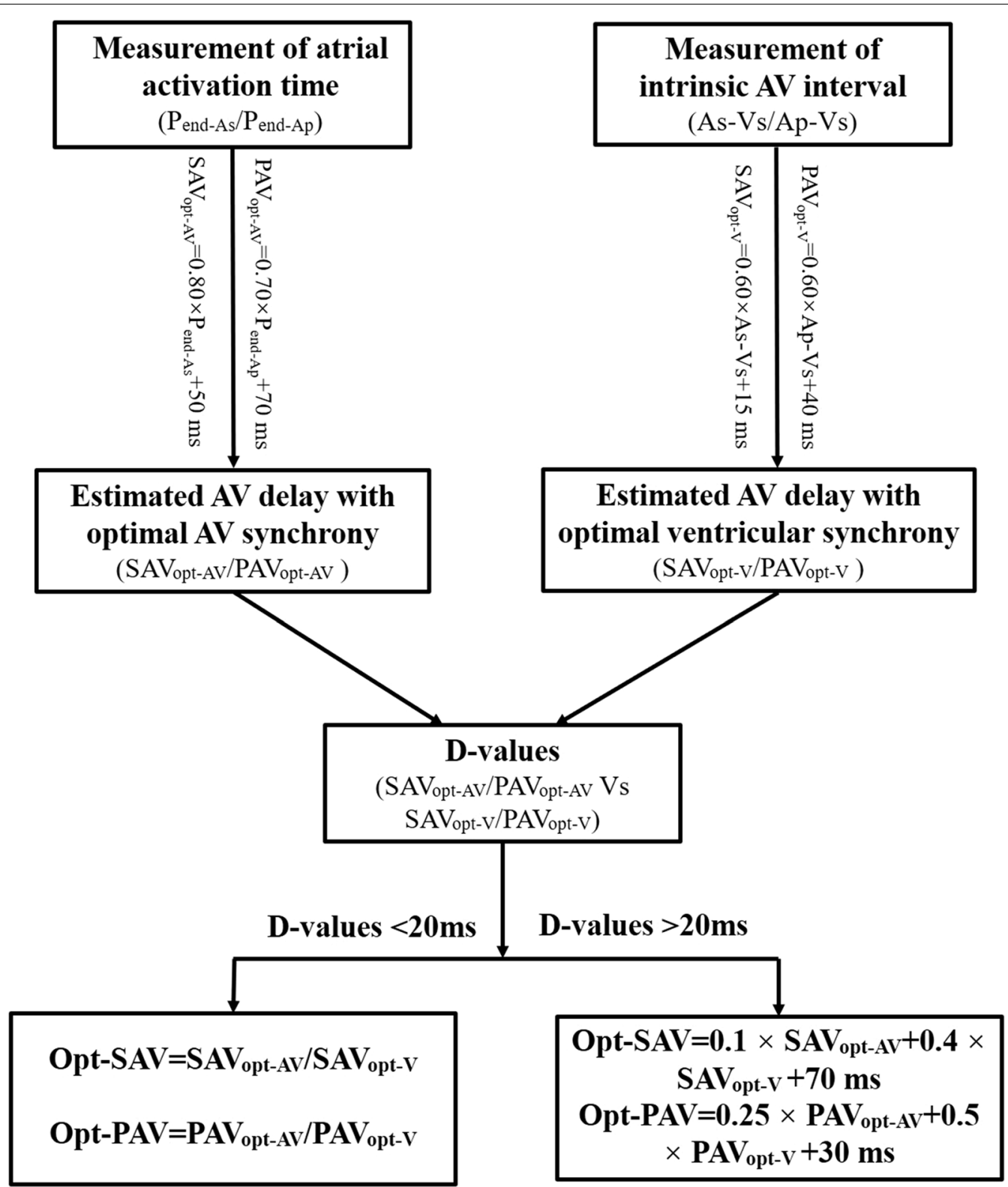

Fig. 4 The optimizing algorithm of the optimal AV delay. $P_{\text {end-As }} / P_{\text {end-Ap: }}$ atrial activation time at atrial sensing/ pacing; $A V$ : atrioventricular; As-Vs/ Ap-Vs: intrinsic atrioventricular conduction time at atrial sensing/pacing; SAV opt-AV/PAV opt-AV: sensing/pacing AV delay optimized according to optimal AV synchrony; $S A V_{\text {opt-V }} / P A V_{\text {opt-v }}$ : sensing/pacing AV delay optimized according to optimal ventricular synchrony; D-values: different values; opt-SAV/opt-PAV: optimal sensing/pacing AV delay with maximal integrative AV and ventricular resynchronization

as other methods (e.g., Doppler-derived AoVTI) [2, 18, 20]. This further indicates that the optimization of the AV delay only according to either optimal AV or ventricular synchrony is not sufficient in CRT. Therefore, the optimal AV delay with maximal hemodynamics improvement should be the AV interval that produces maximal integration of atrioventricular and ventricular synchrony. In this study, we found that the optimal AV delay with maximal
AoVTI was linearly correlated with $\mathrm{SAV}_{\text {opt-AV }} / \mathrm{PAV}_{\text {opt-AV }}$ and $\mathrm{SAV}_{\text {opt-V }} / \mathrm{PAV}_{\text {opt-V }}$ when the D-values between them were $>20 \mathrm{~ms}$. The AoVTIs on opt-AV were either significantly greater or not less than those on $\mathrm{AV}_{\mathrm{opt}-\mathrm{AV}}$ and $\mathrm{AV}_{\text {opt-V}}$. These results indicated that the AV optimization according to the maximal integrative effects of AV and ventricular resynchronization was significantly more effective than the AV interval determined by either optimal AV or ventricular synchrony alone. 
There are several limitations to our study. First, we only enrolled 49 patients with CRT. This relatively small number of cases might have affected the accuracy of the regression formula, although the derived equations were statistically significant. Second, the atrial electrodes were all placed in the right atrial appendages in our patients. The sites of the atrial electrodes could affect the measurement of the atrial activation time (As- $\left.\mathrm{P}_{\text {end }} / \mathrm{Ap}-\mathrm{P}_{\text {end }}\right)$ and the intrinsic $\mathrm{AV}$ conduction time (As-Vs/Ap-Vs). Therefore, the formulas that were used in our study might not be appropriate for other CRT patients whose atrial electrodes were placed in different sits. Third, the location of the LV lead could affect the QRS fusion pattern with intrinsic atrioventricular activation, and was likely to have significant contributions to the $\mathrm{AV}_{\text {opt- } \mathrm{V}}$. Although LV leads were implanted in the middle or basic segment of lateral/posterolateral veins in majority of our cases (65.3\%), it would be better to perform subgroup analysis according to the location of the LV lead and further work is preferable with a sufficient number of cases. Additionally, we only focused on the optimal AV delay in the condition of biventricular simultaneous pacing but did not study the situation of only LV pacing. However, when or how to select the pacing mode of only LV pacing is still controversial. AV and ventricular synchrony must also be considered simultaneously when the AV delay is optimized in only LV pacing mode. Finally, our study was just designed to establish an optimized method with a cross-sectional study, and did not follow the regular visits. Moreover, the detections were performed in patients with stable status of heart failure, which could be seen by the lower using of diuretic in the study cohort. Although the AoVTI was the maximum in the opt-AV, and it is well known that acute hemodynamic improvements measured by echocardiography are related with the outcomes of CRT. The clinical benefit of this algorithm needs further investigation in controlled and prospective studies.

\section{Conclusions}

AV delay could affect atrioventricular and ventricular synchrony in CRT. The AV delay optimized according to the optimal atrioventricular synchrony or optimal ventricular synchrony is correlated with the atrial activation time or the intrinsic AV conduction interval, respectively. However, almost half of the patients showed a significant difference between $\mathrm{AV}_{\text {opt-AV }}$ and $\mathrm{AV}_{\text {opt-V}}$. Optimal $\mathrm{AV}$ delay is the maximal integration of atrioventricular and ventricular synchrony, and could be considered as $\mathrm{SAV}_{\text {opt-AV }} / \mathrm{PAV}_{\text {opt-AV }}$ or $\mathrm{SAV}_{\text {opt-V }} / \mathrm{PAV}_{\text {opt-V }}$ if the D-values were $<20 \mathrm{~ms}$, or could be obtained by formulas that linearly correlated with $A V_{\text {opt-AV }}$ and $A V_{\text {opt- } V}$ when the D-values were $>20 \mathrm{~ms}$.

\section{Abbreviations}

CRT: Cardiac resynchronization therapy; SAV: Sensing atrioventricular delay; PAV: Pacing atrioventricular delay; $\mathrm{AV}_{\text {opt-AV: }}$ AV delay with optimal atrioventricular synchrony; $A V_{\text {opt- }-\mathrm{V}}$ : AV delay optimal ventricular synchrony; opt-AV: The optimal AV delay with the maximum integrative effects of $A V$ and ventricular resynchronization; As: Atrial sensing; Ap: Atrial pacing; Vs: Ventricular sensing; $A s-V s$ : The interval from As to Vs; Ap-Vs: The interval from Ap to Vs; $P_{\text {end-As: }}$ Atrial activation time (duration from As to the end of the $P$ wave); $P_{\text {end-Ap }}$ : Atrial activation time (duration from Ap to the end of the P wave); AoVTI: Aortic velocity-time integral.

\section{Acknowledgements}

None.

\section{Authors' contributions}

$J L, Y W, J M$ and SC were major contributors to the tests performed on the patients directly. ML and CS conducted the statistics analysis. XC, HH, YM, CF, $\mathrm{JJ}$, and Jun Liu assisted with the tests and data collection. JH, AT, YD and XH assisted in the study design and interprettation of the patients. YC and LW were involved in the study design and writing of the manuscript. All authors read and approved the final manuscript.

\section{Funding}

Not applicable.

Availability of data and materials

The datasets could be available in the website: https://pan.baidu.com/s/1kQjx nUNu3pgBdGsC9QliiQ, and the enter code: 7qzz.

\section{Declarations}

Ethics approval and consent to participate

The protocol of the study was approved by Medical Ethics Committee at Sun Yat-Sen University and Nanfang Hospital of Nanfang Medical University. All patients gave written informed consents.

Consent for publication

All authors have reviewed and agree with the content of the article for publication.

\section{Competing interests}

The authors declare that they have no competing interests.

\section{Author details}

${ }^{1}$ Department of Cardiology, The First Affiliated Hospital of Sun Yat-Sen University, No. 58, Zhongshan 2nd Rd, Guangzhou 510080, Guangdong, People's Republic of China. ${ }^{2}$ Key Laboratory On Assisted Circulation, Ministry of Health, Guangzhou, People's Republic of China. ${ }^{3}$ Department of Cardiology, Sun Yat-Sen Memorial Hospital of Sun Yat-Sen University, No.107, Yanjianxi Rd, Guangzhou 510080, Guangdong, People's Republic of China. ${ }^{4}$ Department of Cardiology, Nanfang Hospital of Southern Medical University, Guangzhou, People's Republic of China.

Received: 7 February 2021 Accepted: 3 June 2021

Published online: 10 June 2021

\section{References}

1. Cleland JG, Daubert JC, Erdmann E, Freemantle N, Gras D, Kappenberger $L$, Tavazzi $L$. The effect of cardiac resynchronization on morbidity and mortality in heart failure. N Engl J Med. 2005;352:1539-49.

2. Bristow MR, Saxon LA, Boehmer J, Krueger S, Kass DA, De Marco T, Carson P, DiCarlo L, DeMets D, White BG, DeVries DW, Feldman AM. Cardiacresynchronization therapy with or without an implantable defibrillator in advanced chronic heart failure. N Engl J Med. 2004;350:2140-50.

3. Pires LA, Abraham WT, Young JB, Johnson KM. Clinical predictors and timing of New York Heart Association class improvement with cardiac resynchronization therapy in patients with advanced chronic heart failure: results from the Multicenter InSync Randomized Clinical Evaluation 
(MIRACLE) and Multicenter InSync ICD Randomized Clinical Evaluation (MIRACLE-ICD) trials. Am Heart J. 2006;151:837-43.

4. Jaffe LM, Morin DP. Cardiac resynchronization therapy: history, present status, and future directions. Ochsner J. 2014;14:596-607.

5. Auricchio A, Stellbrink C, Block M, Sack S, Vogt J, Bakker P, Klein H, Kramer A, Ding J, Salo R, Tockman B, Pochet T, Spinelli J. Effect of pacing chamber and atrioventricular delay on acute systolic function of paced patients with congestive heart failure. The Pacing Therapies for Congestive Heart Failure Study Group. The Guidant Congestive Heart Failure Research Group. Circulation. 1999;99:2993-3001.

6. van Gelder BM, Bracke FA, Meijer A, Pijls NH. The hemodynamic effect of intrinsic conduction during left ventricular pacing as compared to biventricular pacing. J Am Coll Cardiol. 2005;46:2305-10.

7. Sweeney MO, Hellkamp AS, van Bommel RJ, Schalij MJ, Borleffs CJ, Bax JJ. QRS fusion complex analysis using wave interference to predict reverse remodeling during cardiac resynchronization therapy. Heart Rhythm. 2014;11:806-13.

8. Ritter P, Padeletti L, Gillio-Meina L, Gaggini G. Determination of the optimal atrioventricular delay in DDD pacing. Comparison between echo and peak endocardial acceleration measurements. Europace. 1999;1:126-30.

9. Cleland JG, Daubert JC, Erdmann E, Freemantle N, Gras D, Kappenberger L, Klein W, Tavazzi L. The CARE-HF study (CArdiac REsynchronisation in Heart Failure study): rationale, design and end-points. Eur J Heart Fail. 2001:3:481-9.

10. Choudhuri I, MacCarter D, Shaw R, Anderson S, St CJ, Niazi I. Clinical feasibility of exercise-based A-V interval optimization for cardiac resynchronization: a pilot study. Pacing Clin Electrophysiol. 2014:37:1499-509.

11. Bogaard MD, Meine M, Tuinenburg AE, Maskara B, Loh P, Doevendans PA. Cardiac resynchronization therapy beyond nominal settings: who needs individual programming of the atrioventricular and interventricular delay? Europace. 2012:14:1746-53.

12. Arbelo E, Tolosana JM, Trucco E, Penela D, Borras R, Doltra A, Andreu D, Acena M, Berruezo A, Sitges M, Mansour F, Castel A, Matas M, Brugada J, Mont L. Fusion-optimized intervals (FOI): a new method to achieve the narrowest QRS for optimization of the AV and $\mathrm{V}$ intervals in patients undergoing cardiac resynchronization therapy. J Cardiovasc Electrophysiol. 2014;25:283-92.
13. Khaykin Y, Exner D, Birnie D, Sapp J, Aggarwal S, Sambelashvili A. Adjusting the timing of left-ventricular pacing using electrocardiogram and device electrograms. Europace. 2011;13:1464-70.

14. Jones RC, Svinarich T, Rubin A, Levin V, Phang R, Murillo J, Sambelashvili A. Optimal atrioventricular delay in CRT patients can be approximated using surface electrocardiography and device electrograms. J Cardiovasc Electrophysiol. 2010;21:1226-32.

15. Hsing JM, Selzman KA, Leclercq C, Pires LA, McLaughlin MG, McRae SE, Peterson BJ, Zimetbaum PJ. Paced left ventricular QRS width and ECG parameters predict outcomes after cardiac resynchronization therapy: PROSPECT-ECG substudy. Circ Arrhythm Electrophysiol. 2011:4:851-7.

16. Linde C, Abraham WT, Gold MR, Daubert C. Cardiac resynchronization therapy in asymptomatic or mildly symptomatic heart failure patients in relation to etiology: results from the REVERSE (REsynchronization reVErses Remodeling in Systolic Left vEntricular Dysfunction) study. J Am Coll Cardiol. 2010;56:1826-31.

17. Tamborero D, Mont L, Sitges M, Silva E, Berruezo A, Vidal B, Delgado V, Tolosana JM, Godoy M, Castel A, Brugada J. Optimization of the interventricular delay in cardiac resynchronization therapy using the QRS width. Am J Cardiol. 2009:104:1407-12.

18. Tamborero D, Vidal B, Tolosana JM, Sitges M, Berruezo A, Silva E, Castel M, Matas M, Arbelo E, Rios J, Villacastin J, Brugada J, Mont L. Electrocardiographic versus echocardiographic optimization of the interventricular pacing delay in patients undergoing cardiac resynchronization therapy. J Cardiovasc Electrophysiol. 2011;22:1129-34

19. Gold MR, Niazi I, Giudici M, Leman RB, Sturdivant JL, Kim MH, Yu Y, Ding J, Waggoner AD. A prospective comparison of AV delay programming methods for hemodynamic optimization during cardiac resynchronization therapy. J Cardiovasc Electrophysiol. 2007;18:490-6.

20. Sawhney NS, Waggoner AD, Garhwal S, Chawla MK, Osborn J, Faddis MN. Randomized prospective trial of atrioventricular delay programming for cardiac resynchronization therapy. Heart Rhythm. 2004;1:562-7.

\section{Publisher's Note}

Springer Nature remains neutral with regard to jurisdictional claims in published maps and institutional affiliations.
Ready to submit your research? Choose BMC and benefit from:

- fast, convenient online submission

- thorough peer review by experienced researchers in your field

- rapid publication on acceptance

- support for research data, including large and complex data types

- gold Open Access which fosters wider collaboration and increased citations

- maximum visibility for your research: over $100 \mathrm{M}$ website views per year

At BMC, research is always in progress.

Learn more biomedcentral.com/submissions 